\title{
STIFFNESS AND STRENGTH OF HIERARCHICAL POLYCRYSTALLINE MATERIALS WITH IMPERFECT INTERFACES
}

\section{MARCO PAGGI ${ }^{1}$ AND PETER WRIGGERS}

\author{
Leibniz University Hannover \\ Institute of Continuum Mechanics \\ Appelstraße 11, 30167 Hannover, Germany \\ e-mail: paggi@ikm.uni-hannover.de, wriggers@ikm.uni-hannover.de
}

Key words: Hierarchical materials; Polycrystalline materials; Imperfect interfaces; Cohesive zone models; Finite element method.

\begin{abstract}
In this paper, considering a cohesive zone model (CZM) for finite thickness interfaces recently proposed by the authors, the stiffness of polycrystalline materials with imperfect interfaces is characterized. Generalized expressions for the Voigt and Reuss estimates of the effective elastic modulus of the composite are derived to interpret the numerical results. Considering a polycrystalline material with a hierarchical microstructure, the interaction between interfaces at the different hierarchical levels is numerically investigated. A condition for scale separation, which suggests how to design the optimal microstructure to maximize the material tensile strength is determined. An original interpretation of this phenomenon based on the concept of flaw tolerance is finally proposed.
\end{abstract}

\section{INTRODUCTION}

The upper and lower bounds to the effective stiffness of a composite material have long been the subject of research (see [1] for an overview). Bounds to the effective properties of heterogeneous solids were put forward by Voigt [2] and Reuss [3]. Eshelby [4] obtained a compact solution that has been the basis for many approximation methods. Based on variational principles, Hashin and Shtrikman [5] developed a refined model for the computation of the effective properties. Several other models have been proposed to estimate the effective properties and their bounds, including computational homogenization [6] for linear and also nonlinear elasticity $[7,8]$. Recent progress in this field regards the analysis of random components, coupling homogenization methods with a statistical description of randomness [9].

\footnotetext{
${ }^{1}$ On leave from Dept. of Structural and Geotechnical Engineering, Politecnico di Torino, Torino, Italy.
} 
Based on Hill's work [10], the effective elastic modulus of a polycrystalline material with perfectly bonded interfaces, $E_{\text {eff }}$, is bounded as follows:

$$
E_{\mathrm{R}} \leq E_{\text {eff }} \leq E_{\mathrm{V}}
$$

where the indices $R$ and $V$ stand for Reuss and Voigt estimates. The Reuss estimate corresponds to isostress conditions, whereas the Voigt estimate is related to isostrain conditions and are given by:

$$
\begin{aligned}
& E_{\mathrm{R}}=\left(\sum_{i=1}^{n} \frac{v_{i}}{E_{i}}\right)^{-1}, \\
& E_{\mathrm{V}}=\sum_{i=1}^{n} v_{i} E_{i},
\end{aligned}
$$

where the summation is extended to all the $n$ material components and $v_{i}$ denotes the volumetric fraction of the $i$-th component $\left(\sum_{i=1}^{n} v_{i}=1\right)$. However, these estimates apply in case of perfectly bonded interfaces, a situation which is often violated in engineering applications.

In this paper, finite element simulations of polycrystalline materials with imperfect interfaces governed by the cohesive zone model (CZM) are carried out. To interpret the numerical results, a generalization of the Voigt and Reuss estimates in case of imperfect cohesive interfaces is proposed. In the second part of the paper, considering a polycrystalline material with a hierarchical microstructure, the effect of interfaces at the different hierarchical levels on the material tensile strength is investigated. The condition for scale separation, often put forward in homogenization methods, is carefully checked and it is found that it is not always fulfilled. When scale separation holds, the material microstructure becomes flaw tolerant and the material tensile strength is maximized, for given interface characteristics at the upper level. These results are useful for a better understanding of the mechanisms leading to the superior mechanical properties of hierarchical polycrystalline materials with respect to their conventional counterpart [11].

\section{GENERALIZED VOIGT AND REUSS ESTIMATES OF THE EFFEC- TIVE ELASTIC MODULUS IN CASE OF IMPERFECT INTERFACES}

To understand how imperfect interfaces modify the bounds to the effective elastic modulus of a heterogeneous material, let us consider the composite layouts shown in Fig. 1. We assume that $m$ material layers behave linear elastically and the remaining $q=n-m$ layers are finite thickness interfaces undergoing damage. Damage is related to the deformation of the interface layer as proposed in [12]. Hence, an effective damage $D_{i}$ is introduced for a generic interface layer $i$ :

$$
D_{i}=\left[\left(\frac{w_{i}}{w_{c, i}}\right)^{2}+\left(\frac{u_{i}}{u_{c, i}}\right)^{2}\right]^{\alpha_{i} / 2}, \quad 0 \leq D_{i} \leq 1
$$




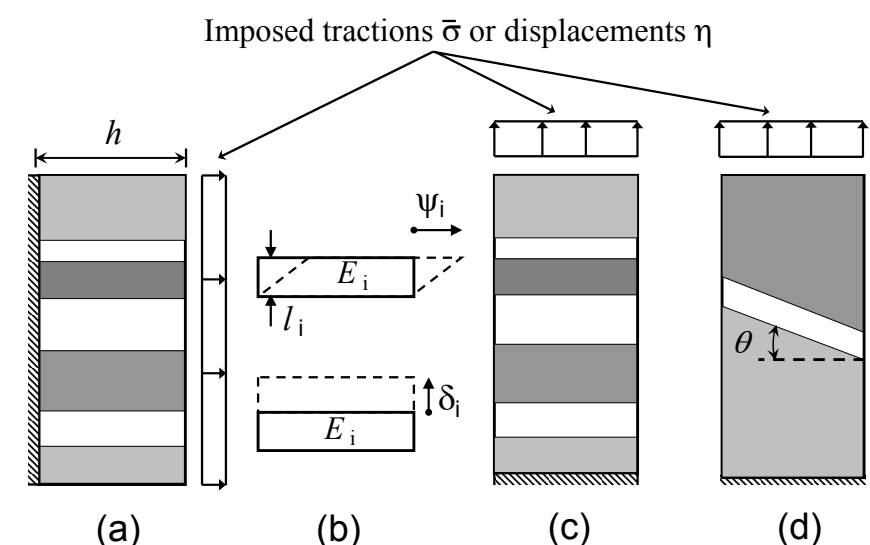

Figure 1: A composite system subjected to imposed tractions or displacements with different orientations of the interfaces with respect to the load direction.

where $w_{i}=\delta_{i}-\delta_{e, i}>0$ is the difference between the displacement of the $i$-th layer in the direction perpendicular to the interfaces (Fig. 1(b))and a threshold value $\delta_{e, i}$ corresponding to the onset of damage. The variable $u_{i}=\psi_{i}-\psi_{e, i}>0$ is the difference between the displacement of the $i$-th layer in the direction parallel to the material interfaces (Fig. $1(\mathrm{~b}))$ and its threshold value $\psi_{e, i}$. The parameters $w_{c, i}$ and $u_{c, i}$ are critical values of the separations in the two orthogonal directions. For more details about the effect of the parameter $\alpha_{i}$ to the evolution of damage, the reader is referred to [12].

Considering the system in Fig. 1(a), isostrain conditions are simulated by imposing uniform horizontal displacements $\eta$ on the vertical boundary on the right and fully restraining the opposite boundary. The horizontal boundaries are traction-free. The horizontal axial strain in each layer equals the average strain $\bar{\epsilon}=\eta / h$, where the distance $h$ is quoted in Fig. 1(a). The stresses are different in the material regions and the average stress is:

$$
\bar{\sigma}=\sum_{i=1}^{n} v_{i} \sigma_{i}=\left(\sum_{i=1}^{n} v_{i} E_{i}\right) \bar{\epsilon}=\left(\sum_{i=1}^{n} v_{i} E_{i}-\sum_{i=1}^{q} v_{i} D_{i} E_{i}\right) \bar{\epsilon},
$$

where the elastic modulus of the damaged interfaces is $E_{i}^{D}=\left(1-D_{i}\right) E_{1}$. A generalized Voigt estimate considering damage in the finite thickness interfaces is therefore derived:

$$
E_{\mathrm{V}}^{\mathrm{G}}=\sum_{i=1}^{n} v_{i} E_{i}-\sum_{i=1}^{q} v_{i} D_{i} E_{i}
$$

For $D_{i}=0$, the classical Voigt estimate for the elastic modulus is recovered. However, neglecting Poisson's effects, it is interesting to note that the boundary conditions for this problem lead to $\delta_{i}=0$ and $\psi_{i}=0$. Consequently, no damage develops and the classical Voigt estimate holds without any modification. 
Let us now consider the same problem but subjected to isostress conditions. They are obtained by imposing a constant horizontal traction $\sigma=\bar{\sigma}$ on the vertical boundary on the right (see Fig. 1(a)). The axial strain is different in the layers and its average is:

$$
\bar{\epsilon}=\left(\sum_{i=1}^{n} \frac{v_{i}}{E_{i}}+\sum_{i=1}^{q} \frac{v_{i} D_{i}}{E_{i}\left(1-D_{i}\right)}\right) \bar{\sigma} .
$$

The effective elastic modulus gives the generalized Reuss estimate considering damage in the finite thickness interfaces:

$$
E_{\mathrm{R}}^{\mathrm{G}}=\left(\sum_{i=1}^{n} \frac{v_{i}}{E_{i}}+\sum_{i=1}^{q} \frac{v_{i} D_{i}}{E_{i}\left(1-D_{i}\right)}\right)^{-1} .
$$

The classical Reuss estimate is recovered when $D_{i}=0$. In this case, however, we cannot exclude a positive relative tangential displacement $u_{i}$ and therefore $D_{i}>0$ in some finite thickness interface.

A different direction of loading with respect to the layer assembly can also be analyzed (see Fig. 1(c)). Vertical displacements or tensile tractions are imposed on the top horizontal boundary. The opposite boundary is fully constrained and the lateral sides are traction free. It is easy to verify that in this case both imposed displacement and imposed traction boundary conditions lead to an isostress state. The mechanical system can be idealized as a set of elastic springs (with damage) in series, whose effective stiffness can be estimated using the generalized Reuss formula (7). In this case, damage is related to a positive displacement $w_{i}$.

The generalized Reuss estimate derived in this study can also be applied to the case of zero-thickness interfaces governed by a standard cohesive zone model (CZM). The additional contribution of the interfaces to the Reuss estimate is due to the displacement discontinuities $g_{T}$ and $g_{N}$ in the tangential and normal directions, respectively. In case of Fig. 1(a) with isostress boundary conditions, the displacement discontinuity (gap) is $g_{T}$ and we have:

$$
\bar{E}=\left(\sum_{i=1}^{n} \frac{v_{i}}{E_{i}}+\sum_{i=1}^{q} \frac{g_{T, i}}{h}\right)^{-1} .
$$

For the case in Fig. 1(c), the displacement discontinuity is represented by $g_{N}$ :

$$
\bar{E}=\left(\sum_{i=1}^{n} \frac{v_{i}}{E_{i}}+\sum_{i=1}^{q} \frac{g_{N, i}}{h}\right)^{-1} .
$$

For an interface inclined by a generic angle $\theta_{i} \neq 90^{\circ}$ with respect to the direction of loading (see Fig. 1(d)), mixed mode deformation occurs and both $w_{i}$ and $u_{i}$ contribute to $D_{i}$. For a standard CZM, Eq.(9) should be used, with $g_{N, i}$ determined in correspondence of a given angle of mode mixity, $\theta_{i}=\arctan \left(g_{T, i} / g_{N, i}\right)$. 


\section{STIFFNESS OF HIERARCHICAL POLYCRYSTALLINE MATERIALS}

In this section, the stiffness of a cellular polycrystalline material with imperfect interfaces is numerically analyzed using the finite element method. One forth of a representative volume element (RVE) of the hexagonal microstructure is shown in Fig. 2(a). Zero-thickness interface elements are introduced between the linear elastic constant strain triangular elements used to discretize the continuum. The constitutive response of the interfaces is governed by the nonlocal CZM for finite thickness interfaces proposed in [12]. An implicit solution scheme is adopted, which requires the computation of the tangent stiffness matrix and the residual vector of the interface elements to be used in a Newton-Raphson iterative scheme. Moreover, the treatment of the CZM requires a nested Newton-Raphson loop to compute the cohesive tractions, see [13]. The RVE is tested in uniaxial tension, imposing the displacements on the nodes belonging to the vertical boundary on right.

Different bulk moduli $E_{\mathrm{B}}$ for the grains are considered. For infinitely stiff grains, $E_{\mathrm{B}} \rightarrow \infty$, the deformability is only due to the cohesive interfaces. The obtained dimensionless stress-strain curve is therefore that of the CZM of the inclined interface with a rescaled abscissa $g_{N} / h$, where $h$ is the horizontal lateral size of the sample (see Fig. $2(\mathrm{~b})$ ). Reducing $E_{\mathrm{B}}$, the deformability of the continuum increases and the effect of the interfaces is mitigated. The nonlinear CZM is responsible for the deviation from linearity in the stress-strain diagram, as it can be quantified by comparing the solid lines with the corresponding dashed lines in Fig. 2(b).

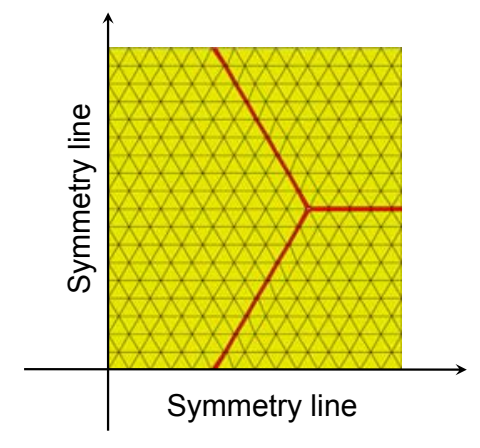

(a) $1 / 4$ of the RVE.

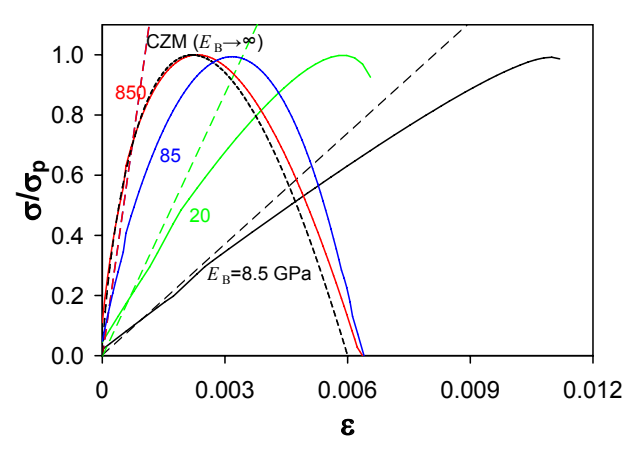

(b) Average stress vs. strain response.

Figure 2: The effect of the bulk modulus $E_{\mathrm{B}}$ on the average stress-strain response.

The computed tangent effective elastic modulus vs. $\sigma / \sigma_{p}$, where $\sigma_{p}$ is the peak stress, is shown in Fig. 3(a). The CZM response is the upper limit for $E_{\text {eff }}$, obtained for infinitely stiff grains. The curves become progressively independent of $\sigma / \sigma_{p}$ by reducing $E_{\mathrm{B}}$. Since the specimen is subjected to isostress conditions, the obtained trend can be interpreted according to Eq.(7) with $n=1$ and $q=1$. Using the shape of the CZM corresponding to the angle of mode mixity induced by the hexagonal geometry $\left(\theta=30^{\circ}\right)$, the analytical 
predictions are shown in Fig. 3(b). The perfect agreement with the numerically computed curves in Fig. 3(a) is achieved.

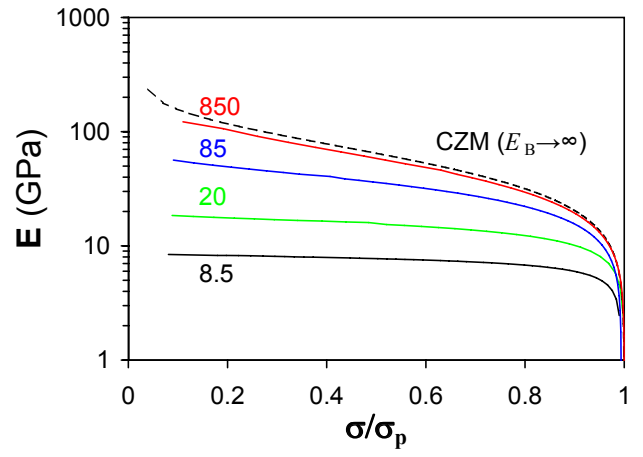

(a) Numerical results.

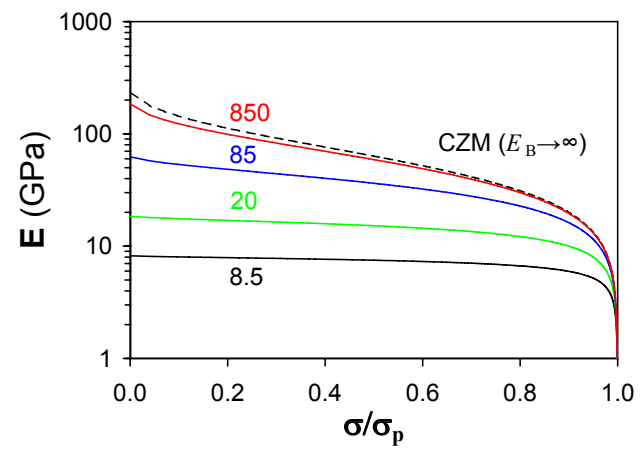

(b) Analytical predictions.

Figure 3: Tangent effective elastic modulus $E$ vs. $\sigma / \sigma_{p}$

Two further levels of hierarchy are now considered. The macroscopic level $(l=1)$ is the same as that analyzed in Fig. 2. Each grain is then partitioned into other cellular grains, generating the material mesostructure $(l=2)$. This procedure is repeated once more to obtain the material microstructure $(l=3)$ inside the mesogranules, see Fig. 4(a). For the sake of simplicity, and without any loss of generality, the CZM parameters are assumed to be the same at each level. The refinement of the material microstructure leads to a decrease of the dimensionless effective elastic modulus $E / E_{\mathrm{B}}$ for a given stress level, see Fig. 4(b). The numerical predictions using Eq.(7) with a constant angle of mode mixity equal to $30^{\circ}$ are still a good approximation of the numerical results. More accurate predictions can be obtained by considering the actual angle of mode mixity of the various interfaces.

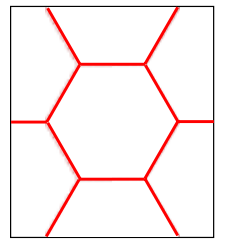

$l=1$

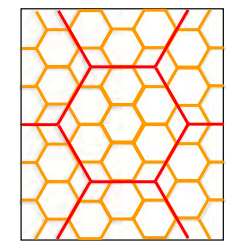

$l=2$

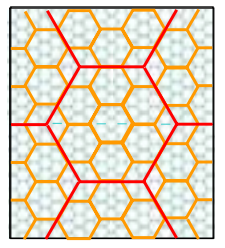

$l=3$

(a) Sketches of the hierarchical levels.

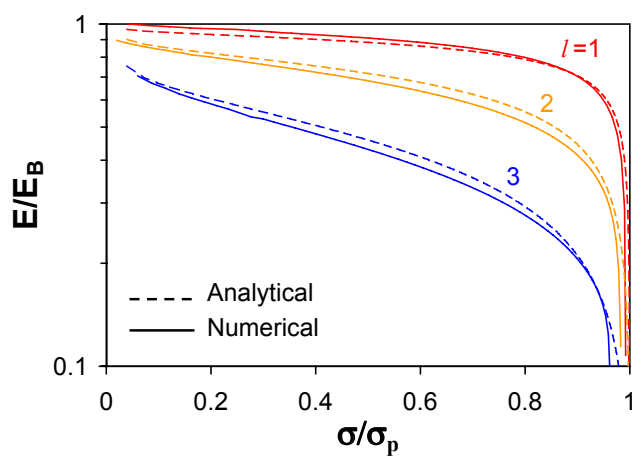

(b) Numerical-analytical comparison.

Figure 4: Tangent effective elastic modulus $E / E_{\mathrm{B}}$ vs. $\sigma / \sigma_{p}$ for a system with $l$ hierarchical levels. 


\section{STRENGTH OF HIERARCHICAL POLYCRYSTALLINE MATERIALS}

In this section we study focus on the effect of interfaces with different properties on the tensile strength of hierarchical polycrystalline materials. Let us consider the finest microstructure with hexagonal grains examined in the previous section as the level 1 (blue interfaces in Fig. 5(a)). A two-level hierarchical microstructure is realized by considering geometrically self-similar hexagonal mesogranules (level 2, red interfaces in Fig. 5(a)) embedding the hexagonal micrograins of the level 1. To do so, the interface characteristics at the level 2 are modified in order to make them tougher than those at the level 1 . This condition alone is not sufficient to modify the crack pattern at failure and improve the material response. In fact, it is still possible to find a subvertical crack path involving only interfaces of level 1 , as it happens without hierarchy. To involve the interfaces of the level 2, all the properties of the interfaces of the hexagons containing a triple junction have to be modified. This leads to the final configuration shown in Fig. 5(a).

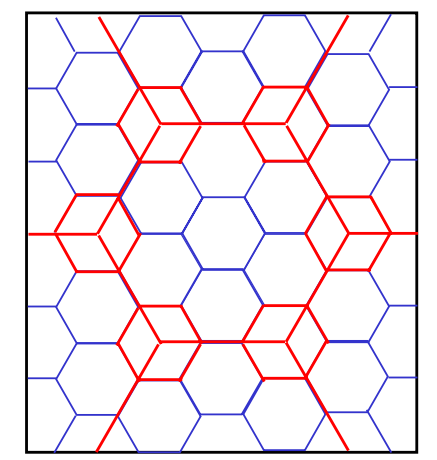

(a) Interfaces at the 2 levels.

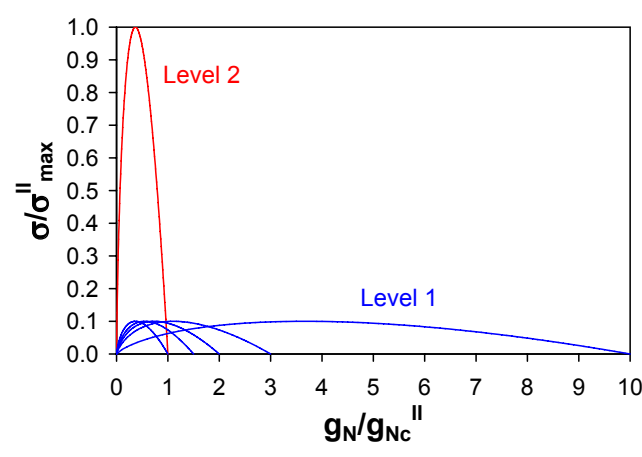

(b) Shape of the CZMs.

Figure 5: CZMs of the interfaces at the different levels: level 1 (blue), level 2 (red).

The material response is now investigated by performing virtual tensile tests in the horizontal directions and computing the peak stress $\sigma_{p}$ of the average stress-strain curve. The shapes of the Mode I CZMs of the interfaces at the levels 1 and 2 are shown in Fig. 5(b). The ratio between the peak cohesive tractions of the CZMs at the two levels is constant and equal to $\sigma_{\max }^{\mathrm{I}} / \sigma_{\max }^{\mathrm{II}}=0.1$. Different Mode I fracture energies (evaluated as the area between the Mode I CZM curve) for the level 1 are considered, exploring a range $G_{\mathrm{IC}}^{\mathrm{I}} / G_{\mathrm{IC}}^{\mathrm{II}}$ from 0.1 to 0.5 (see the different shapes of the CZM of the level 1 in Fig. 5(b)).

Dimensional analysis considerations suggest the following dependency for the tensile strength $\sigma_{p}$ on the variables of the parametric analysis:

$$
\sigma_{p}=\sigma_{p}\left(\sigma_{\max }^{\mathrm{I}}, G_{I C}^{\mathrm{I}}, d^{\mathrm{II}}, E_{\mathrm{B}}\right),
$$

where $\sigma_{\max }^{\mathrm{I}}$ is the peak cohesive traction at the level $1, G_{\mathrm{IC}}^{\mathrm{I}}$ is the Mode I fracture energy of the interfaces at the same level, $d^{\mathrm{II}}$ is the diameter of the mesogranules and $E_{\mathrm{B}}$ is the 
bulk elastic modulus. Considering $G_{\mathrm{IC}}^{\mathrm{I}}$ and $d^{\mathrm{II}}$ as the variables with independent physical dimensions, the application of the Buckingham's theorem of dimensional analysis yields:

$$
\frac{\sigma_{p} d^{\mathrm{II}}}{G_{\mathrm{IC}}^{\mathrm{I}}}=\Phi\left(\frac{\sigma_{\max }^{\mathrm{I}} d^{\mathrm{II}}}{G_{\mathrm{IC}}^{\mathrm{I}}}, \frac{E_{\mathrm{B}} d^{\mathrm{II}}}{G_{\mathrm{IC}}^{\mathrm{I}}}\right)=\Phi\left(s_{1}, s_{2}\right) .
$$

Changing $G_{\mathrm{IC}}^{\mathrm{I}}$, the dimensionless numbers $s_{1}$ and $s_{2}$ vary at the same time. It is therefore convenient to introduce a combination of these dimensionless numbers and plot the results of the parametric analysis as a function of it. Considering $s=s_{2} / s_{1}^{2}$, we have:

$$
s=\frac{s_{2}}{s_{1}^{2}}=\frac{G_{\mathrm{IC}}^{\mathrm{I}} E_{\mathrm{B}}}{\left(\sigma_{\max }^{\mathrm{I}}\right)^{2} d^{\mathrm{II}}}=\frac{l_{\mathrm{CZM}}^{\mathrm{I}}}{d^{\mathrm{II}}} .
$$

This dimensionless number is proportional to the ratio between the critical process zone size at the level $1, l_{\text {CZM }}^{\mathrm{I}}$, and the grain size of the mesograin, $d^{\mathrm{II}}$.

From numerical results (Fig. 6), the computed dimensionless tensile strength is an increasing function of $l_{\mathrm{CZM}}^{\mathrm{I}} / d^{\mathrm{II}}$ up to $l_{\mathrm{CZM}}^{\mathrm{I}} / d^{\mathrm{II}}=1$, when a plateau is reached. A further increase of the interface fracture energy of the level 1 does not permit us to increase the tensile strength. This result can be explained according to a consideration of flaw tolerance, as also proposed by Gao [14] for defects in bone-like materials. When the interface cracks inside the mesogranule (having a size $a \leq d^{\mathrm{II}}$ ) are such that $l_{\mathrm{CZM}}^{\mathrm{I}}>$ $d^{\mathrm{II}}$, their maximum opening displacement lies within the range of cohesive interactions. Therefore, no stress-free cracks develop in the mesogranule, which is able to tolerate the intrinsic defects of any size. Fracture has to involve the upper scale interfaces at the level 2 and the strength is maximized. Similar considerations have been put forward by Carpinteri [15] for the problem of tension collapse that precedes brittle crack propagation in a strip. These results are important for the application of homogenization techniques. In fact, a separation of scales, leading to no interaction between the interfaces at the different hierarchical levels is fulfilled only for $l_{\mathrm{CZM}}^{\mathrm{I}} / d^{\mathrm{II}}>1$.

The diagram in Fig. 6 can also be interpreted in a different way. For given material interface properties at the level 1 ( $l_{\mathrm{CZM}}^{\mathrm{I}}$ is fixed), the optimal geometry of the mesostructure corresponds to $d^{\mathrm{II}}=l_{\mathrm{CZM}}^{\mathrm{I}}$. In this way, the interfaces at the upper level act as crack arresters for the microcracks at the lower level. When this flaw tolerance condition is achieved at a given level, the same reasoning can be repeated for the higher hierarchical levels in order to obtain the optimal response for a given set of material properties.

\section{CONCLUSION}

In the present paper, the role of imperfect interfaces on the stiffness and strength of hierarchical polycrystalline materials has been numerically investigated using finite elements. The constitutive behavior of finite thickness interfaces has been modelled using the nonlocal CZM recently proposed in $[12,13]$.

Numerical simulations show that the effective elastic modulus depends on the debonding process occurring at the interfaces. Generalized Voigt and Reuss estimates accounting 


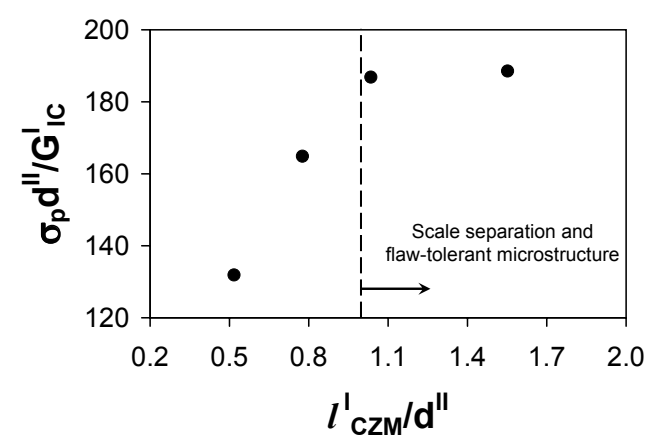

Figure 6: Dimensionless tensile strength vs. ratio between the critical process zone size and the diameter of the mesogranules.

for such a form of nonlinearity are proposed for the computation of the bounds to the tangent effective elastic modulus. The interplay between the interface deformability and the elasticity of the bulk material has been numerically characterized.

Finally, the effect of interfaces at different hierarchical levels on the strength of a twolevel hierarchical material has been numerically investigated. The results show that the condition for scale separation applies only for certain properties of the interfaces at the lower level. If such a condition, represented by the inequality $l_{\mathrm{CZM}}^{\mathrm{I}}>d^{\mathrm{II}}$, is fulfilled, then the microstructure becomes flaw tolerant and the strength is ruled by the interfaces characteristics at the upper level.

\section{ACKNOWLEDGEMENTS}

MP would like to thank the Alexander von Humboldt Foundation for supporting his research stay at the Leibniz Universität Hannover during the year 2010.

\section{REFERENCES}

[1] Nemat-Nasser, S. and Hori, M. Micromechanics: Overall Properties of Heterogeneous Solids. Elsevier, Amsterdam (1999).

[2] Voigt, W. Über die Beziehung zwischen den beiden Elastizitätskonstanten isotroper Körper. Wied. Ann. (1889) 38:573-587.

[3] Reuss, A. Berechnung der Fliessgrenz von Mischkristallen auf Grund der Plastizitätsbedingung fur Einkristalle. Z. Angew. Math. Mech. (1929) 9:49-58.

[4] Eshelby, J.D. The elastic field of an ellipsoidal inclusion, and related problems. Proc. Royal Soc. A (1957) 241:376-396.

[5] Hashin, Z., Shtrikman, S. On some variational principles in anisotropic and nonhomogeneous elasticity. J. Mech. Phys. Solids (1962) 10:335-342. 
[6] Zohdi, I.I., Wriggers, P. Introduction to Computational Micromechanics. Springer, Berlin, Heidelberg, New York (2005).

[7] Temizer, İ., Zohdi, T.I. A numerical method for homogenization in non-linear elasticity. Comp. Mechanics (2006) 40:281-298.

[8] Temizer, İ., Wriggers, P. An adaptive method for homogenization in orthotropic nonlinear elasticity. Comp. Meth. Appl. Mech. Engng. (2007) 196:3409-3423.

[9] Ma, J., Temizer, İ., Wriggers, P. Random homogenization analysis in linear elasticity based on analytical bounds and estimates. Int. J. Solids and Struct. (2011) 48:280291.

[10] Hill, R. The elastic behavior of a crystalline aggregate. Proc. Phys. Soc. London A (1952) 65:349-354.

[11] Fang, Z.K. et al. Fracture resistant super hard materials and hardmetals composite with func-tionally graded microstructure. Int. J. Refr. Met. \& Hard Mat. (2001) 19:453-459.

[12] Paggi, M., Wriggers, P. A nonlocal cohesive zone model for finite thickness interfaces Part I: mathematical formulation and validation with molecular dynamics. Comp. Materials Science (2011) 50:1625-1633.

[13] Paggi, M., Wriggers, P. A nonlocal cohesive zone model for finite thickness interfaces Part II: FE implementation and application to polycrystalline materials. Comp. Materials Science (2011) 50:1634-1643.

[14] Gao H. Application of fracture mechanics concepts to hierarchical biomechanics of bone and bon-like materials. Int. J. Fract. (2006) 138:101-137.

[15] Carpinteri A. Notch sensitivity in fracture testing of aggregative materials. Engng. Fract. Mech. (1982) 16:467-481. 